\title{
CREEP IN THE EARTH AND PLANETS
}

\author{
(Invited Lecture) \\ HAROLD JEFFREYS \\ St. John's College, Cambridge, England
}

\begin{abstract}
An outstanding problem is to reconcile the Moon's rotation with the persistence of its nonhydrostatic dynamical ellipticities. The first requires imperfection of elasticity under strains of order $10^{-7}$; the second apparently little under larger ones.

Lomnitz gave experimental evidence that creep is linear at elastic shears from $10^{-5}$ to $10^{-4}$, indicating that a linear rule could be right at still smaller values. Positive evidence for the Earth comes from the damping of the 14-monthly nutation, which has a relaxation time of the order of $30 \mathrm{yr}$. Most work on imperfect elasticity has assumed that under constant shear stress the strain increases with time either like $t$ (elasticoviscosity) or like $\log t$. If the result from the 14-monthly nutation, with elasticoviscosity, is applied to the Moon, the dynamical ellipticities would have subsided considerably in the last $200 \mathrm{yr}$. With the logarithmic rule an $S$ pulse at $80^{\circ}$ would have its beginning spread out over about $70 \mathrm{~s}$ and be unreadable. These contradictions are avoided if the increase under constant stress is about like $t^{0.2}$. The resulting law involves two constants. Without change of these, applications are made to other phenomena. The rotations of the Moon and of other satellites whose rotations are known are explained; so is the persistence of the Moon's dynamical ellipticities; also the failure to detect three free oscillations that might theoretically exist. Elasticoviscosity would imply rapid disappearance of the non-hydrostatic second and third harmonics in the Earth's gravitational field; this is avoided with the new law. Study of damping of free vibrations of the Earth (including surface waves) has usually assumed the logarithmic law, but it appears that the new law fits the data at least as well, and that it may also explain those that have been interpreted in favour of layers of low velocity. It appears that the damping at depths up to $400 \mathrm{~km}$ or so is much more severe than the average for the Earth's shell, and more evidence for shorter periods is much needed.

Any law with an index less than 1 would forbid thermal instability (convection) and continental drift.
\end{abstract}

The subject goes back to Darwin's papers in the 1880's on tidal friction as an explanation of the observed secular acceleration of the Moon. Darwin had no quantitative data on imperfections of elasticity in the Earth (and in fact very few on the ordinary elasticity). He generally treated the Earth as a liquid of high viscosity; but he worked out the theory properly and most of his results can be adapted to other imperfections of elasticity. He mentions a law suggested by Maxwell and put into mathematical equations by J. G. Butcher, and called elastico-viscosity. According to this, if $P$ is the shear stress applied at $t=0$ and kept constant, and $\varepsilon$ the shear, they are related by

$$
\varepsilon=\frac{P}{\mu}\left(1+\begin{array}{l}
t \\
\tau
\end{array}\right)=\frac{P}{\mu}\left(1+\frac{1}{p \tau}\right)
$$

in Heaviside's notation. The fundamental definition is that if $f(t)=0$ for $t<0$,

$$
\frac{1}{p} f(t)=\int_{0}^{t} f(\tau) \mathrm{d} \tau \text {. }
$$

Many generalizations are possible; these are in Jeffreys and Jeffreys (1966), Chapters 7, 8 and 12. For $\tau$ infinite this is perfect elasticity. For $\mu$ tending to $\infty$ while $\mu \tau \rightarrow \eta$ 
it gives ordinary viscosity. For more general cases, if a problem has been solved for perfect elasticity, the solution for elastico-viscosity can be derived by replacing $\mu$ by the operator $\mu /(1+1 / p \tau)=\mu p \tau /(p \tau+1)$. In particular if there is a time factor $e^{i \gamma t}, p$ must be replaced by $i \gamma$. For large $\gamma$ the behaviour approximates to perfect elasticity; for small $\gamma$ (long periods) to viscosity.

The immediate application was to tidal friction. For a perfect fluid or a perfectly elastic solid the tidal elevations will be just in line with the disturbing body; but with any dissipation of energy they will be after these places have passed these points. Then the forces on the projections will give a couple tending to slow the rotation. The immediate application is to the fact that the Moon keeps a constant face to the Earth. The tides raised in the Earth by the Moon would also tend to slow the Earth's rotation. The couple reacting on the Moon increases its energy, which is accommodated by making the Moon recede. The two effects together lead to an apparent secular acceleration when the Earth's rotation is used to give a standard of time. (Incidentally, since the couple is the result of the Moon's attraction on a tide itself raised by this attraction, it is a second-order effect, which however can be calculated from a first-order theory.)

The qualitative position was satisfactory; but it led to the position that whenever a body was found to be rotating more slowly than might be expected people said 'tidal friction' and left it at that. The first attempt to check it quantitatively by other evidence was in a paper of mine in 1915. The Earth's axis has a free oscillation in a period of about 14 months, predicted by Euler. It was discovered by Chandler after Darwin's papers. If the Earth was perfectly rigid the period would be about 10 months, and the difference is due to elasticity. If the Moon's secular acceleration is attributed to tidal friction, we can get an estimate of the quantity $\tau$ in the elastico-viscous law. But applying this to the free nutation gave a time of relaxation of about 5 days. Something was clearly wrong.

Another check was provided by the figure of the Moon. The differences between its principal moments of inertia are determined from forced oscillations of the directions of its principal axes, and were shown by Laplace to be about 16 times what they would be in a fluid body. But if the Moon is imperfectly elastic these should be subsiding. I showed that the facts could be reconciled if the Moon last adjusted itself to a hydrostatic state when its period of revolution was about 6 days and thenceforward remained perfectly elastic. The difficulty about tidal friction in the Earth was largely resolved by G. I. Taylor's discovery of the importance of turbulent currents in the Irish Sea, and my extension to other shallow seas, which showed that these currents could produce enough dissipation in the semidiurnal tide to account for the secular acceleration of the Moon. Their effect on the free nutation would be negligible. So no evidence remained for imperfection of elasticity in either the body of the Earth or in the Moon now.

Evidence emerged however in an analysis of the observations of the free nutation, which at first indicated a time of relaxation of about $15 \mathrm{yr}$, though the statistical problem of eliminating the forced annual period and separating the disturbances that keep the motion going was difficult. Recent work using observations over 60 years revises this to about $30 \mathrm{yr}$, but the uncertainty remains large. 
There had long been experimental evidence that in solids the creep under small constant stress does not increase as $t$, but as $\log t$ or possibly $t^{1 / 3}$. These had the obvious fault that they make $\mathrm{d} \varepsilon / \mathrm{d} t \rightarrow \infty$ as $t \rightarrow 0$, which does not happen even for a perfectly elastic body. But C. Lomnitz noticed three important things in rocks, one theoretical and the other two experimental. If the law is taken to be

$$
\varepsilon={ }_{\mu}^{P}[1+q \log (1+a t)]
$$

with $q$ and $a$ constant, $\mathrm{d} \varepsilon / \mathrm{d} t$ remains finite as $t \rightarrow 0$; and for large $t$ the creep is like $\log t$. For such stresses that the initial strain $P / \mu$ is from $10^{-5}$ to $10^{-4}$ the stress-strain relation was linear, suggesting that a linear relation can be extrapolated to smaller stresses; and $a$ was so large that if $t$ is $1 \mathrm{~s} a t$ swamps 1 . This had actually been noticed by Phillips in 1903 in other materials but forgotten. In tides and the variation of latitude the strains are of order $10^{-6}$ to $10^{-7}$; also for seismic waves at $\Delta>10^{\circ}$ or so.

Applying this to the damping of the free nutation gave an estimate of $q$, but it could also be applied to an $S$ wave emerging at about $80^{\circ}$, taking about $20^{\mathrm{m}}$ to travel. The result was that the beginning would be spread out over about $70^{\mathrm{s}}$ and be quite unreadable. But there was an easy remedy. If we modify the power law similarly we get

$$
\varepsilon={ }_{\mu}^{P}\left[1+{ }_{\alpha}^{q}\left\{(1+a t)^{\alpha}-1\right\}\right]
$$

with behaviour for large $t$ like $t^{\alpha}$. I call this the modified Lomnitz law. It has interesting consequences. If $\alpha=1$ the creep becomes $(P / \mu) q$ at, so that it varies with time as for elasticoviscosity. As $\alpha \rightarrow 0$ it tends to the logarithmic law. For a forced vibration of period $T$ the lag is as $T^{\alpha}$, tending to a constant as $\alpha \rightarrow 0$. The amplitude of a wave travelling a wave length contains an exponent as (period) ${ }^{\alpha}$; one travelling a given distance one as (period) $)^{-1+\alpha}$, with a factor depending on distance alone. Thus if $\alpha<1$ there will be a tendency for shorter periods to be damped out. In addition if there is a time factor $e^{i \gamma t}$, for pure elasticity, the disturbance at distance $x$ will be expi $(t-x / \beta)$. Here we must replace $1 / \beta$ by

$$
\frac{1}{\beta_{0}}\left(1+\frac{q a^{\alpha}}{2 \alpha} \frac{\alpha !}{(i \gamma)^{\alpha}}\right)=\frac{1}{\beta_{0}}\left\{1+\frac{q a^{\alpha} \alpha !}{2 \alpha \gamma^{\alpha}}\left(\cos \frac{1}{2} \pi \alpha-i \sin \frac{1}{2} \pi \alpha\right)\right\} .
$$

The imaginary part gives a negative exponent proportional to $x$, and hence the damping; but the real part gives also an imaginary part $\cot \frac{1}{2} \pi \alpha$ times the negative one. Thus there is also a systematic effect on times of transmission when $\alpha<1$. It is only for elasticoviscosity that a mixture of periods can travel without change of the ratios of their amplitudes. There is an analogous effect on free periods, which will show a damping but also a lengthening.

The creep depends essentially on two parameters, $\alpha$ and $q a^{\alpha}$. The free nutation gives 
one equation for these; the reasonable hypothesis from the look of $S$ that an $S$ pulse at $80^{\circ}$ reaches half its final value in $2^{\mathrm{s}}$ gives another. For a unit pulse at $x=0, t=0$ the displacement is

$$
\begin{aligned}
& \frac{1}{2 \pi i} \int_{L} \exp \lambda\left(t-\frac{x}{\beta}\right) \frac{\mathrm{d} \lambda}{\lambda} \\
& =H(t-x / \beta) .
\end{aligned}
$$

$\beta$ is the purely elastic velocity, $v(\mu / \varrho)$. The important change for free vibrations, with the new law, is that $1 / \beta$ has to be replaced by

$$
\frac{1}{\beta_{0}}\left(1+\frac{q a^{\alpha}}{2 \alpha} \frac{\alpha !}{p^{\alpha}}\right)
$$

in Heaviside's form.

Then the displacement $I(v)$ takes the form

$$
I(v)=\frac{1}{2 \pi i} \int_{L} \exp v\left(u-\frac{u^{1-\alpha}}{1-\alpha}\right) \frac{\mathrm{d} u}{u}
$$

with

$$
v=\left(t-\frac{x}{\beta_{0}}\right)^{(\alpha-1) / \alpha}\left(\frac{1}{2} q a^{\alpha}(\alpha-1) ! \frac{x}{\beta_{0}}\right)^{1 / \alpha}(1-\alpha)^{1-\alpha}
$$

This looks horrid, but some things follow easily. There is no displacement till time $x / \beta_{0}$; and as $t \rightarrow \infty, I \rightarrow 1$, so that the final result is as for perfect elasticity. For intermediate values of $t$ there is nothing for it but heavy arithmetic, which Stuart Crampin did for me. The result is that $\alpha$ is probably between 0.14 and 0.21 , with 0.19 as the most probable value. $q a^{\alpha}$ would be $0.00054 /\left(1^{s}\right)^{\alpha}=0.0142 /\left(1^{y r}\right)^{\alpha}$. An $S$ wave at $80^{\circ}$ has been nearly down to the core and the 14-monthly period affects all the Earth's shell, so these can be taken as average properties of the shell.

We can now study other effects of the law. First take the subsidence of a surface inequality. Suppose a uniform incompressible gravitating sphere of radius $b$ to be loaded at time 0 with matter of uniform density to depth $b \varepsilon_{0} S_{n}$. The elastic displacement is $b \varepsilon_{1} S_{n}$, where

$$
\varepsilon_{1}\left(1+\frac{n b \varrho g}{\left(2 n^{2}+4 n+3\right) \mu}\right)=-\frac{n b \varrho g \varepsilon_{0}}{\left(2 n^{2}+4 n+3\right) \mu}
$$

The total height above the mean sphere is $b\left(\varepsilon_{0}+\varepsilon_{1}\right) S_{n}$. Write

$$
\varepsilon_{0}+\varepsilon_{1}=\varepsilon^{\prime}, \quad \frac{n}{2 n^{2}+u n+3}=N, \quad\left(1+\frac{N b \varrho g}{\mu}\right) \varepsilon^{\prime}=\varepsilon_{0} .
$$


To adapt to creep we modify this to

$$
\varepsilon^{\prime}=\frac{\varepsilon_{0}}{1+\frac{N b \varrho g}{\mu}\left(1+\frac{(\alpha-1) ! q a^{\alpha}}{p^{\alpha}}\right)} H(t)
$$

Expansion in negative powers of $p$ gives a convergent series. We find that for the Earth, if $n=2$ and $t=10^{8} \mathrm{yr}$, the subsidence would be of order $50 \%$ (15\% to large). For an elevation of wave length $10^{8} \mathrm{~cm}$ the losses would be $1.6,10$ and $22 \%$ according as $\alpha$ is taken as $0.14,0.19$, or 0.21 . The result for $n=2$ is consistent with O'Keefe's interpretation of the difference between the Earth's actual and hydrostatic ellipticities as being due to the last adjustment to the hydrostatic state having been when the rotation was faster. Wave length $10^{8} \mathrm{~cm}$ is of the order of the width of mountain systems; and the subsidence is too small to account for isostasy. But this is really irrelevant because the elastic strains are of order $10^{-3}$ and it is just here that a linear stress-strain relation must fail. For pure viscosity the results are quite different. If an anomaly $100 \mathrm{~km}$ in horizontal extent has had time to be halved, one of $3000 \mathrm{~km}$ would be reduced to $10^{-9}$ of its original amount. But the greatest discoveries from artificial satellites are the uncompensated second and third harmonics in the gravitational field. With elasticoviscosity, if higher ones have been reduced at all, these low ones should have disappeared.

For the Moon the losses of a harmonic of degree 2 in $10^{8}$ yr would similarly be 1,5 and $11 \%$; in $3 \times 10^{9} \mathrm{yr}, 2,10$ and $23 \%$. There is no difficulty in understanding the persistence of the dynamical ellipticities. But if we choose $\tau$ in the elasticoviscous law to fit the damping of the free nutation, we find that they should have decreased substantially in the last $200 \mathrm{yr}$, the interval of reasonably good observation.

The effect of tidal friction on rotation is treated in various places. The angular acceleration is

$$
\dot{\omega}=-{ }_{4}^{45}\left(\frac{n^{2} a}{1+\kappa}\right)^{2} \frac{\varrho}{19 \mu_{0}+2 g \varrho a} \sin 2 \varepsilon
$$

where $\omega$ is the rate of rotation, $n$ that of revolution and $2 \varepsilon$ the lag of the semidiurnal tide. Other quantities refer to the disturbed body. $\kappa$ expresses the ratio of the body's rotational and orbital angular momenta; for all cases except the Earth it is small. What we can notice at once is that the most important factor is $-\left(n^{2} a\right)^{2}$. Comparison for different satellites shows that it is larger (for some much larger) in comparison with the Moon for Phobos, Deimos, the four great satellites of Jupiter, those of Saturn out to Titan, and the old ones of Uranus and Neptune. In fact all the satellites whose rotations are known keep constant faces to their primaries, which is what we should expect if their properties are anything like those of the Moon. However there is an oddity about Iapetus, for which this factor is much less than for the Moon and which certainly keeps a constant face to Saturn. There may well be some difference in its composition that makes the lag or the elastic yield larger. 
Mercury has a long period of rotation, though recent work indicates that it is $\frac{2}{3}$ of the period of revolution and not equal to it. The lags for periods up to 100 days are from 0.004 to 0.010 in circular measure. The time intervals starting from a period of $12 \mathrm{hr}$ to reach the following synodic rotation periods are as follows:

$\begin{array}{ll}\text { Period (days) } & \text { Time (years) } \\ 0.5 & 7 \times 10^{9} \\ 1 & 3 \times 10^{9} \\ 2 & 1.7 \times 10^{9} \\ 20 & 0.1 \times 10^{9} \\ 200 & \end{array}$

These are of the right order of magnitude but the total is larger than we should expect.

For the Moon the changes are much more rapid on account of the factor $n^{4}$. There is no difficulty in accounting for the rotation of the Moon.

Incidentally no planet beyond the Earth can have had its rotation greatly altered by bodily tidal friction, and no planet at all can have had its orbital period greatly altered.

The Moon's axes have theoretically three periods of free oscillation in direction, with periods of about 1,1600 and 40 months. With the same constants they would have times of relaxation of the order of $10^{6} \mathrm{yr}$, so that there is no difficulty in understanding why they have not been found. There has been no claim that the first two have been detected. Some recent work has claimed to detect the third, but the period and phase are in close agreement with a forced term with an argument twice the distance between the Moon's node and perigee, and the observations probably refer to the latter.

Much information on other lines has been derived from the damping of seismic waves (especially surface waves) and free vibrations. The theoretical periods of free vibrations are systematically longer than those observed, by about $1 \%$. Landisman et al. (1965) infer a surprising constancy of density in the lower part of the shell (from depth $1500 \mathrm{~km}$ to the core boundary); Bullen and Haddon (1967) an increase of about $20 \mathrm{~km}$ in the radius of the core. My 1940 estimate of the core radius had an apparent standard error of $3.5 \mathrm{~km}$. It was a bit indirect as $S c S$ was compared with $S$ and $S$ with $P$, and these comparisons bring in additional uncertainties, but $I$ think not seriously. Buchbinder (1965) gives observations of $P c P$ and $I$ find that they indicate an increase by $3 \pm 5 \mathrm{~km}$. The times of $S$ are still in some doubt. Up to $25^{\circ}$ or so comparison of

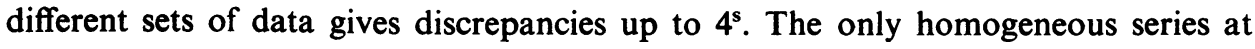
large distances is Arnold's for Japanese deep foci, and Japan is abnormal for $\boldsymbol{P}$. But $4^{\mathrm{s}}$ in $25^{\mathrm{m}}$ is about $\frac{1}{400}$ and is unlikely to be the reason. Alsop, Sutton and Ewing for the slowest free vibration $\left({ }_{0} S_{2}\right)$ give period $53^{\mathrm{m}}, Q=370$. (1/Q is the damping of energy 
$(T+V)$ when the phase increases by a radian.) With distance travelled $3000 \mathrm{~km}$ the damping would be of order $\frac{1}{200}$. It agrees in order of magnitude. But with $\alpha=0.2$ the factor on the period would be about $\frac{1}{130}$. Of the three explanations I think this is the most likely.

The appearance of $\cos \frac{1}{2} \pi \alpha-i \sin \frac{1}{2} \pi \alpha$ in the solutions is a case of the allied Fourier integral. This occurs in the relation between potential and charge function in electrostatics over a cylinder or a plane, to various problems in vibrations, and to the theory of a thin aerofoil. With any linear law of vibrations the allied Fourier integral enters if the amplitude is known for different forced speeds and we want to calculate the phase. The results so far concern average properties of the shell. I worked out the damping of $S$ for different periods with $\alpha=0.2$ and $x / \beta_{0}=200^{\mathrm{s}}$, corresponding to distance about $7^{\circ}$.

$\begin{array}{ll}\text { Period (s) } & k(-k \text { index in amplitude }) \\ 1 & 0.3 \\ 2 & 0.16 \\ 5 & 0.1 \\ 10 & 0.06 \\ 20 & 0.04 \\ 50 & 0.02\end{array}$

Amplitudes of both $P$ and $S$ (first swing) fluctuate by a factor of order 100 in $8^{\circ}<\Delta<20^{\circ}$. These results do not account for this. $5^{\mathrm{s}}$ is a typical period of first swing in $S\left(\log _{e} 100=\right.$ 4.6). To account for the variation $k$ would have to be multiplied by about 50 .. Shimshoni and I showed that it could be explained by a variation of $d^{2} t / \mathrm{d} \Delta^{2}$ (the squared amplitude contains this as a factor) but it requires rather a coincidence. But $\cot \frac{1}{2} \pi \alpha$ is about 3 and the phase shift for $k=5$ would be about 15 radians. For period $5^{\mathrm{s}}$ this gives a delay of $(15 / 2 \pi) \times 5 \doteqdot 15^{\mathrm{s}}$. This is ample to explain layers of low velocity and the large delayed $S$. But the explanation requires my $q a^{\alpha}$ to be much larger than average in a layer about $100 \mathrm{~km}$ down.

This is confirmed by studies of the damping of surface waves. The results are mostly stated in terms of $Q$.

\begin{tabular}{llc}
\hline & Depth & $Q$ \\
Anderson and Archambeau (1964) give & $0-400 \mathrm{~km}$ & 100 \\
& $400-800 \mathrm{~km}$ & 200 \\
& $800-1000 \mathrm{~km}$ & 1000 \\
Anderson et al. (1965) & $1000-$ core km & 2000 \\
& Upper layers & $400 \pm 200$ \\
& $70 \mathrm{~km}$ & $80 \pm 30$ \\
& $100-150 \mathrm{~km}$ & $130 \pm ?$
\end{tabular}

All these assume damping independent of period and effectively imply the logarithmic law. Mostly they find no appreciable variation with period, but the range of periods 
used is from about $10^{\mathrm{s}}$ to $2000^{\mathrm{s}}$, and $(200)^{0.2}$ is about 3 . So it is not surprising that they did not detect variation with period. (I recall that variation with period is needed to explain why $S$ can be read at all.) But Ben-Menahem (1965) gets values that suggest a variation with period, but if they applied to a uniform body they would make $\alpha$ negative. But the longer periods penetrate to greater depths; and the variation can be explained by increase of $q a^{\alpha}$ with depth without change of $\alpha$.

The free nutation extends the range of period greatly. At the other end the periods in $S$ itself are of order $1^{\text {s }}$ at short $\Delta$ and analysis of $S$ might tell us more. The main difficulty is that (especially for surface waves) the shorter the period the less the depth where amplitudes are large; so it may be hard to separate variation with depth from variation with period.

Rayleigh's principle should be most useful in this connexion. Given an approximate solution for a free vibration it will give first-order corrections for any small change of properties. Explicit forms for the required kinetic and potential energies are given by Jeffreys and Vicente (1967). Material for effects of changes of density and elastic properties is given (by longer methods) by Wiggins (1969) and Derr (1969) and could be easily adapted to the modified Lomnitz law.

One point of importance concerns the possibility of convection. For steady convection in a fluid we can write down the equations of motion and heat conduction, and they lead to a differential equation for the disturbance of temperature from adiabatic

$$
\nabla^{6} V^{\prime}=\frac{G}{v} \nabla_{1}^{2} V^{\prime}
$$

$v$ is the kinematic viscosity, $\nabla_{1}^{2}$ is the Laplace operator without the derivatives with regard to $v$. $G$ depends on various parameters of the problem. The thermodynamic argument is given in detail by Jeffreys (1956). For the modified Lomnitz law this has to be modified to (Jeffreys 1958)

$$
\nabla^{6} V^{\prime}=H p\left(1+k p^{-\alpha}\right) \nabla_{1}^{2} V^{\prime} .
$$

This will be correct to the two lowest powers of $p$, and the lowest on the right gives $\nabla^{6} V^{\prime}=H k p^{1-\alpha} \nabla_{1}^{2} V^{\prime}$. Then in the case of $p=0$ (steady motion) and $\alpha<1$ we get $\nabla^{6} V^{\prime}=0$. This has no solutions except $V^{\prime}=0$ for the given boundary conditions. Hence with the law I have been considering, with $\alpha<1$, convection is impossible. Convection depends absolutely on $\alpha$ being 1 ; that is, it requires elasticoviscosity. Physically the reason is as follows. In the viscous case the excess temperature in the heated columns is maintained by warm liquid flowing in at the bottom, and the velocity is maintained by shear stresses. The essential point is that constant shear stress in this case implies constant rate of shear. But with any law with $\alpha<1$, even if the stresses were constant, the rate of shear would tend to zero as time increases. Thus if convection was started it could not continue to bring in enough heat to replace the loss by conduction. Similarly continental drift at a constant rate would need a continual increase of stress, tending to infinity after a long time. 


\title{
References
}

\author{
Alsop, E. E., Sutton, G. H., and Ewing, M.: 1961, J. Geophys. Res. 66, 631. \\ Alsop, E. E., Sutton, G. H., and Ewing, M.: 1961, J. Geophys. Res. 66, 2911. \\ Anderson, D. L. and Archambeau, C. B.: 1964, J. Geophys. Res. 69, 2071. \\ Anderson, D. L., Ben-Menahem, A., and Archambeau, C. B.: 1965, J. Geophys. Res. 70, 1441. \\ Ben-Menahem, A.: 1965, J. Geophys. Res. 70, 4641. \\ Buchbinder, G. G. R.: 1965, Bull. Seism. Soc. Am. 55, 441. \\ Bullen, K. E. and Haddon, R. A. W.: 1967, Nature 213, 574. \\ Derr, J. S.: 1969, Bull. Seism. Soc. Am. 74, 5202. \\ Jeffreys, H.: 1956, Quart. J. Mech. Appl. Math. 9, 1. \\ Jeffreys, H.: 1958, Geophys. J. Roy. Astron. Soc. 1, 162. \\ Jeffreys, H.: 1965, Nature 208, 675. \\ Jeffreys, H. and Jeffreys, B. S.: 1966, Methods of Mathematical Physics, Camb. Univ. Press. \\ Jeffreys, H. and Vicente, R. O.: 1967, Acad. Roy. Belg. Bull. Cl. Sci. (5) 53, 926. \\ Jeffreys, H.: 1970, The Earth, esp. pp. 12-13, 47, 331-334, 490, 494. \\ Jeffreys, H. and Crampin, S.: 1970, Monthly Notices Roy. Astron. Soc. 147, 295. \\ Landisman, M., Sato, Y., and Nafe, J.: 1965, Geophys. J. Roy. Astron. Soc. 9, 439. \\ Wiggins, M. A.: 1968, Phys. Earth Planetary Int. 1, 201.
}

